\title{
Performance Evaluation of a Relocation Method for Mobile Computing Maintaining Data Consistency
}

\author{
Byung-Kwan Lee ${ }^{1}$ and Am-Suk Oh ${ }^{2}$ \\ ${ }^{1}$ Dept. of Computer Engineering, Kwandong Univ. \\ San-7, YangYangyup, YangYanggun, KwangwonDo, Korea, \\ bklee@kwandong.ac.kr \\ ${ }^{2}$ Dept. of Multimedia Engineering, TongMyong University \\ of Information Technology. Yongdang-dong, Nam-gu, Busan, Korea \\ asoh@tmic.tit.ac.kr
}

\begin{abstract}
Recently, a method to use replicated data on a server in order to get new data without missing any information has been studied on wireless telecommunication networks and satellite services. Static Replica Allocation (SRA) that traditional scheme has been used for the replication method on the server. This replicates the data on the replica server after a moving host has been transferred to another cell. In case the number of users is smaller than that of cells, the network of the SRA is an efficient scheme in the standpoint of access cost, and if there are few moving users, no trouble will happen. But if there are no moving users in a cell, the data will not be shared. This paper proposes new method of relocation, that is, USRA (User Select Replica Allocation) based on data consistency scheme (called USRAC) after replicating the data to the cells for the users, and also analyzes access cost according to the moving rate of mobile users, according to the access rate of mobile hosts, and according to the number of cells of mobile users and mobile hosts.
\end{abstract}

\section{Introduction}

Mobile computers and wireless communication networks have made it possible to open up a new field in mobile computing. Mobile computing provides mobile users with the ability to access information regardless of the location of the user or the information. In traditional distributed database systems, various strategies have been suggested in order to process transaction and maintain consistency. But most solutions are restricted to mobile computing because of mobility, network failure and lower bandwidth. The replication of data allows mobile computers to quickly access data. But consistency among duplicated data should be maintained. The distributed database in the mobile computer environment for replicating data has used SRA(Static Replica Allocation). SRA is a scheme that when a mobile host moves to a cell, replicates data to the replica server within the cell. In case the network is stable and a number of mobile users smaller than cells, this scheme does not cause any problem with using data. However, after the mobile host moved to a cell, if no mobile user existed in the 
replicated cell, replicated data become useless. And this scheme is not suitable for the mobile computing environment because of frequent disconnection, lower bandwidth, etc.

In this paper, we present our replica allocation called USRAC (User Selection Replica Allocation based on Consistency scheme) which makes up for defects of SRA scheme and is appropriate to the mobile computer environment. This scheme replicates data and then allocates it to the cell in which mobile users are numerous. We have conducted a detailed performance evaluation of SRA, USRA and USRAC scheme proposed in terms of access cost and communication cost based on access ratio of mobile user, access ratio of mobile host and variation of number of cells.

\section{SRA (Static Replica Allocation)}

Replication is the process that automatically updates data involved in a replica server maintains the same object and data. Whenever mobile users need to replicate data, they can do it. The replication is used to increase the speed of the query and information retrieval. SRA(Static Replica Allocation) is a strategy that has been currently researched. SRA is a method that replicates data to the replica server of moved cell if a mobile user requires data. The advantage of this method is that data replication is simple and easily relocates data. However, because mobile host replicates data to moved cell, in case mobile user does not exist in the moved cell, the replicated data can be deleted for consistency maintenance. And if mobile host leaves replicated cell, the access of data through route becomes difficult because of the frequent disconnection of mobile computing environment

The advantage of SRA strategy is to easily replicate transacted data and replication is simple. Therefore, this scheme is suitable when the network is stable and mobile users in the cell are smaller than mobile support stations. However, the first disadvantage of this scheme is that searching the route of the cell in which data transacted is replicated is difficult. Second, although location replicated is known, it is not easy to access a data through route since a frequent disconnections is occurred. Third, in case mobile user does not exist in the replicated cell, data transacted cannot be used. Fourth, if a mobile host moves, the data of replica server is deleted since the data of replica server cannot maintain consistency.

\section{USRAC (User Selection Replica Allocation Based on Consistency Scheme)}

\subsection{USRA Process}

Because SRA strategy is difficult to trace the location of a replicated data and use a data and maintain consistency, we propose a new replication scheme called USRA (user selection replica allocation). USRA strategy is a scheme that a replica server is 
assigned to a cell in which mobile users are numerous and then relocates a data to the replica server. However, when access cost is computed to this scheme, cost to relocate replication is also added since this strategy additionally should relocate to the cell in which mobile users are more numerous.

USRA is strategy that determines the location relocated of replication at location server and assigns replica server. Replica server takes data information and location information and then provides the data which mobile user requires. This scheme resolves disadvantage that data is not used or the location route of a data has to be searched since selects cell which the user of a data is more numerous and replicates the data. If the relocation of replication is not determined, the loading of data replicated may increase.

\subsection{Data Consistency Scheme}

The data consistency among duplicated data in the mobile computing environment should be maintained. Thus, we present a data consistency scheme for USRA.

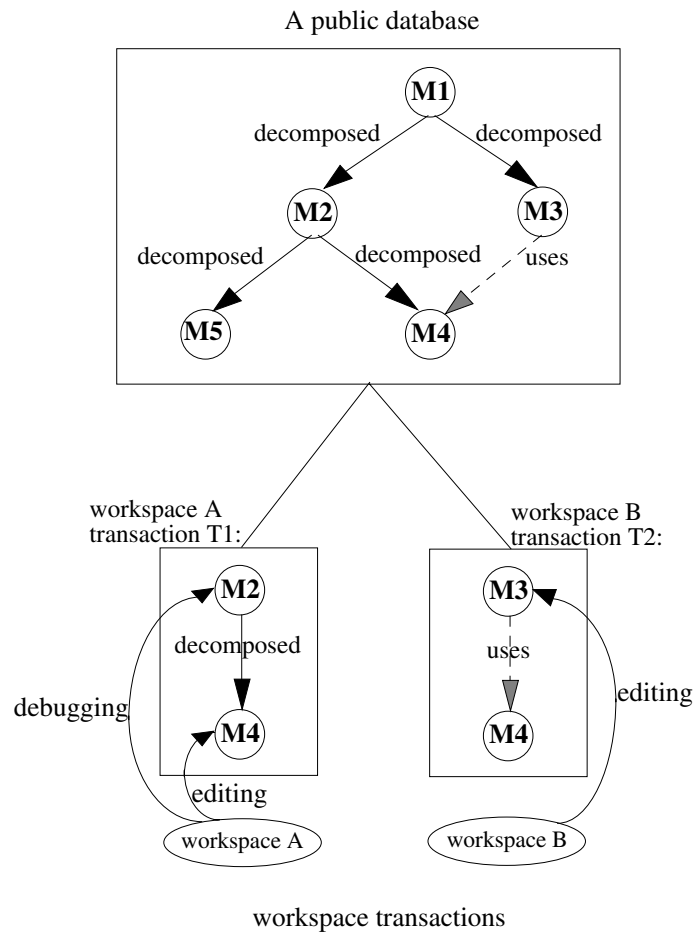

Fig. 1. A Transaction Model 


\subsubsection{Transaction Model}

Fig. 1 shows a transaction model for data consistency. A transaction T1 develops two programs, M2 and M4, at workspace A. The other transaction T2 edits M3 and M4 at workspace B. Here we assume that M2 is decomposed into M4 and M5, where M4 is also used by M3. If M3 uses M4, we also say that M3 is a client of M4, since M3 requires the interfaces that M4 provides. The solid arrow denotes the relationship 'decomposed'. The dashed arrow denotes the relationship 'uses'.

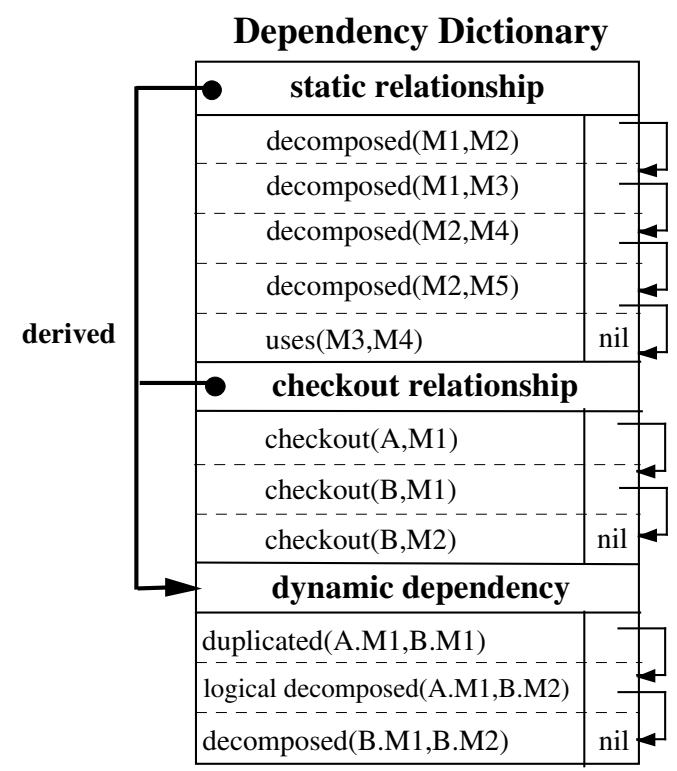

Fig. 2. Consistency Table

\subsubsection{Consistency Table}

To represent the consistency scheme, we use the structure of linked lists, because the dynamic dependency grows and shrinks according to check-in/check-out operations performed. The consistency table shown in Fig. 2, consists of three lists: a list of static relationships, a list of checkout relationships, and a list of dynamic relationships. In Fig. 2, the static relationships represent those relationships among all objects in the public database. For instance, the relationship 'decomposed(M1,M2)' means that M1 is decomposed into M2. The list of static relationships is not influenced by any checkout operation. However, a new static relationship may be added to the list of static relationships by means of checkin operations. 


\subsubsection{Consistency Scheme}

Fig. 3 is a scheme for data consistency. Fig. 3(a) represents the state before M2 is checked out to workspace C. Fig. 3(b) represents the state where M2 has been newly checked out to workspace C. The dashed rectangle within the consistency table in Fig. 3 (b) shows the new relationships generated after M2 is checked out into workspace C.

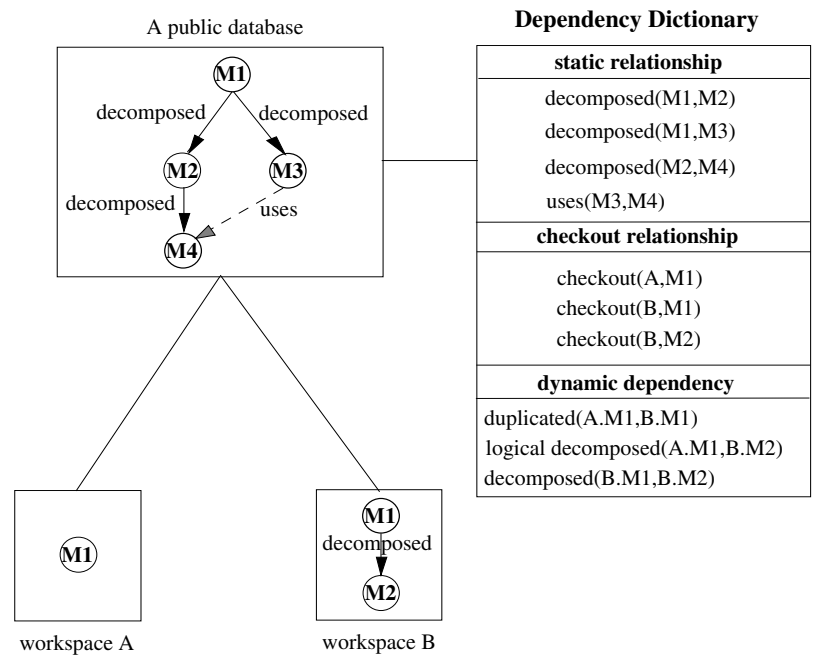

(a) before M2 is checked out

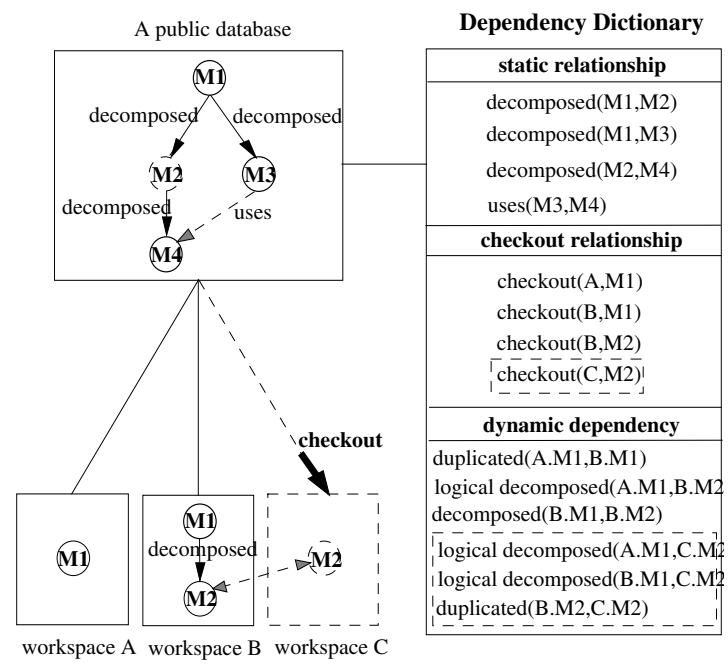

(b) after M2 is checked out

Fig. 3. Scheme for data consistency 


\section{Experiments and Performance Evaluation}

We evaluated performance among SRA, USRA and USRAC strategy in the mobile computing environment by computing access cost and communication cost.

\subsection{The Access Cost for Move Ratio of Mobile User}

Fig. 4 illustrates the access cost of SRA, USRA and USRAC for the variation of access ratio $(\alpha)$ of mobile host. The move ratio of mobile user is generated from 0 to $1 \lambda$. We found out that if $\alpha$ is over 0.8 , access cost maintains as fig. 4 regardless of variation of $\alpha$ value.

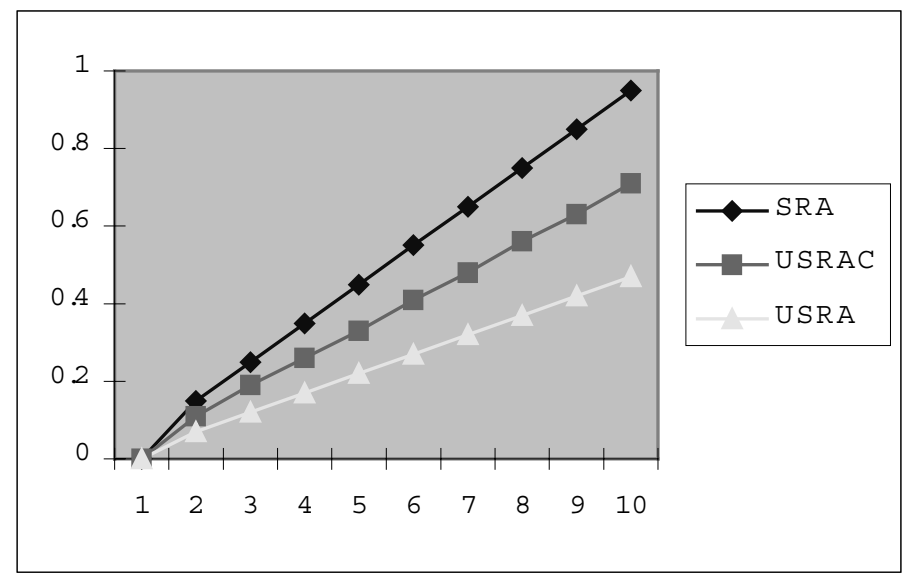

Fig. 4. The access cost of mobile user for $\alpha=0.8$

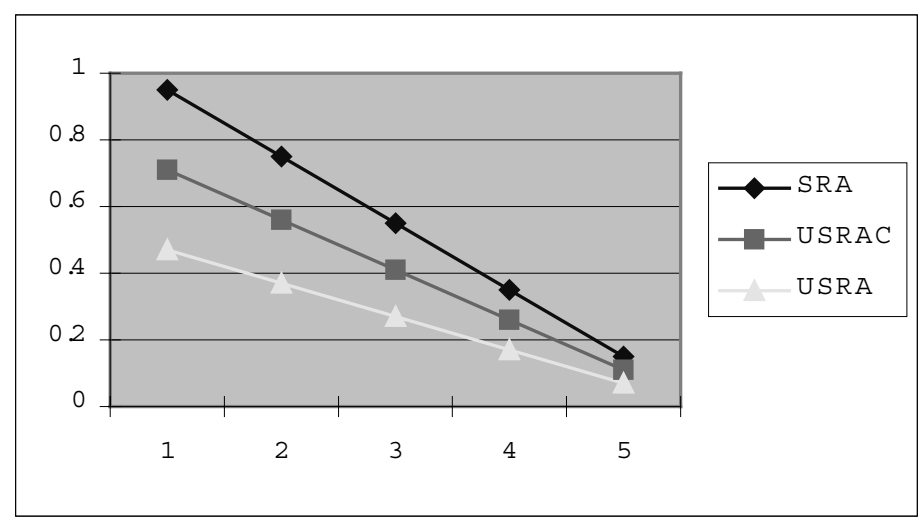

Fig. 5. The access cost of mobile user for $\lambda=0.8$ 


\subsection{The Access Cost for Access Ratio of Mobile Host}

Fig. 5 illustrates the access cost of mobile host as the access ratio $(\lambda)$ of mobile user and the access ratio of mobile host varies from 0 to 1 . When the access $\operatorname{ratio}(\lambda)$ of mobile user is over 0.8 , the result is as fig. 5 .

\subsection{The Access Cost for The Number of Cells}

Fig. 6 illustrates the access cost of SRA, USRA and USRAC for variation of the number of cells. We found out that the access cost of USRA and USRAC is more sensitive than SRA to the variation of the number of cells. Because the replicated cell also increases when the number of cells increases.

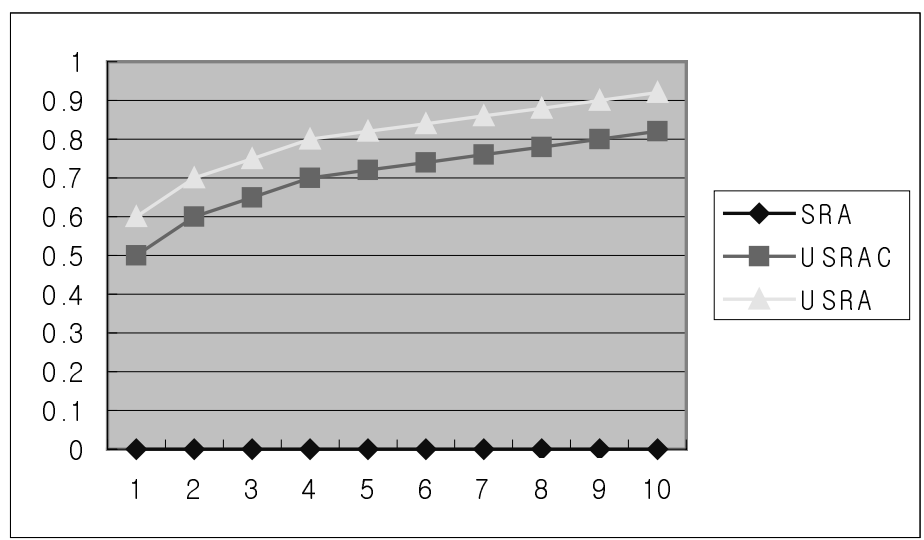

Fig. 6. The access cost for the number of cell to $\alpha=0.8, \lambda=0.8$

\section{Conclusions}

In this paper we have considered USRAC scheme that relocates a replication for mobile computers. SRA(Static Replica Allocation) is a method that replicates a data to the replica server of moved cell if a mobile user requires a data. SRA takes the advantage that data replication is simple and can easily relocate data. However, if mobile user or mobile host is not existed in the moved cell then the replication data becomes useless and it makes move ratio is high. Because USRA selects and replicates the cell that the number of mobile user is most numerous, the move ratio of mobile user can be reduced. However, USRA scheme was not considered serious problem of data consistency in the mobile computing environments. In this paper we have investigated three schemes - SRA, USRA and USRC scheme and have evaluated performance in terms of cost based on schemes. Conclusively, though the USRAC scheme that we have proposed is medium cost, it makes maintain data consistency in the mobile computing. 


\section{References}

1. A. Chandrasekaran and R. W. Broderson, "A portable multimedia terminal for personal communications," IEEE Communications Magazine, pp. 64-75, December 1992.

2. A. V. Bakre and B. R. Badrinath, "Implementation and performance evaluation of indirect TCP," IEEE Trans. on Computer, vol. 46, no. 3, pp. 260-278, March 1997.

3. D. Duchamp, S. K. Feiner, and G. Q. Maguire, Jr., "Software technology for wireless mobile computing," IEEE Nerwork Magazine, pp. 12-18, November, 1991.

4. D. J. Goodman, G. P. Pollini, and K. S. Meier-Hellstern, "Network control for wireless communications," IEEE Communications," IEEE Communications Magazine, pp. 116124, 1992.

5. P. Evaggelia et al., "Revising Transaction Concepts for Mobile Computing", proc. IEEE Workshop on Mobile Systems and Applications, Dec 1994.

6. F. Teraoka, K. Claffy, and M. Tokoro, "Design, implementation, and evaluation of virtual internet protocol," IEEE Distributed Computing Systems DCS'92, pp. 170-177, 1992.

7. G. H. Forman and J. Zahorjan, "The challenges of mobile computing," IEEE Computer, pp. 38-47, April 1994.

8. K. Lim and Y. H. Lee, "Optimal partitioning of heterogeneous traffic sources in mobile communications networks," IEEE Trans. on Computer, vol. 46, no. 3, pp. 312-325, March 1997.

9. K. H. Choi, "Data Relocation Using User Selecting Replica Allocation in Mobile Computing Environment," Phd Thesis, University of Kwandong, 1999.

10. T. Imielinski and B. R. Badrinath, "Mobile wireless computing," Communications of the ACM, vol. 37, no. 10, pp. 18-28, October 1994.

11. T. Imielinski and B. R. Badrinath, "Data Management for mobile computing," the ACM SIGMOD RECORD. vol. 22, no.1, pp. 34-39, 1993.

12. Y. Huang, P. Sistla, and O. Wolfson, "Data replication for mobile computers" Proc. of the 1994 ACM SIGMOD Intl. Conf. on Management of Data, pp. 13-24, 1994. 